\title{
High-Speed Adaptive RF Phased Array
}

\author{
Dimitrios N. Loizos $^{1,2}$, Paul P. Sotiriadis ${ }^{2}$ and Gert Cauwenberghs ${ }^{1}$ \\ ${ }^{1}$ Division of Biological Sciences, University of California, San Diego, CA 92093 \\ ${ }^{2}$ Department of Electrical and Computer Engineering, The Johns Hopkins University, Baltimore, MD 21218
}

\begin{abstract}
We demonstrate dynamic power maximization and synchronization of a wireless RF communication link through adaptation of the radiation pattern of a phased array at the transmitter. Adaptation is performed through a multi-dithering, coherent detection gradient descent analog controller chip [1], and compensates for phase variations in the communication link. The chip, located at the transmitter, controls the phase of each element in the array so that all transmitted signals combine coherently in phase at the receiver. The control objective is the downconverted RF signal at the receiver, which is fed back to the input of the chip through a reverse, lower bandwidth analog RF link. Measurements on the demonstrated prototype, consisting of a 4-element phased array at the transmitter and an omnidirectional receiver, indicate microsecond scale continuous-time optimization of the system.
\end{abstract}

\section{INTRODUCTION}

Beamforming of multiple antenna elements in a phased array allows to optimize the efficiency of wireless communications, by maximizing the power of the signal at the receiver, and hence maximizing signal-to-noise ratio for a given transmitted power [2]. Highly precise and agile beamsteering with phased arrays has been demonstrated for radar applications in free space. In cluttered mobile environments, occlusions and multiple reflections impact the received power, making beamsteering ineffective for power maximization. Hence, indoors configurations typically adopt a sub-optimal omni-directional scheme.

In principle, power can be optimally transmitted over an array by compensating for the net phase shift between transmitter and receiver for each element in the array. For beamsteering in free space, these phase shifts are directly given by the direction cosines of the receiver relative to the transmitter array. In more general environments it becomes more difficult or intractable to compute these phase shifts from the transmitter-receiver geometry. Rather than computing phase shifts, here we consider adaptive maximization of received power, by observing the effect of perturbing the phase shifts on the measured power. By dithering the phase shifts at RF frequencies, we accomplish high bandwidth adaptive power maximization for highly agile mobile communications.

More specifically, adaptation is performed on the downconverted signal at the receiver, which provides a performance metric that links the phases of the received signals to that of the LO (Local Oscillator) at the receiver. As will be shown in Section III, optimization of this performance metric results in phase locking of all received signals to the receiver LO (RLO). This translates to synchronization as well as phase coherent combination of the transmitted signals at the receiver and, thus, power maximization. The system under consideration is illustrated in Fig. 1. The transmitting antennas can be driven by either separate independent LOs or by a common source in a phased array configuration. Without loss of generality, the demonstrated system assumes a single LO at the transmitter side (TLO).

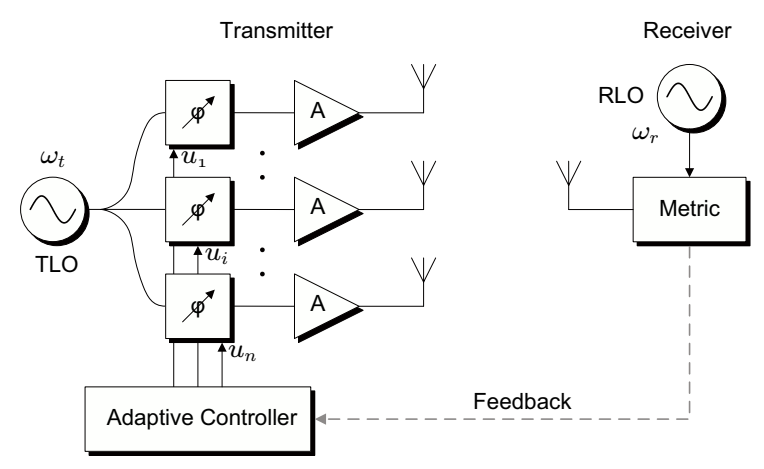

Fig. 1. General architecture of the wireless communication system with adaptive power maximization and synchronization.

\section{Multi-Dithering AdAPTATion}

Coherent combining of the transmitted signals and phase locking of the presented phased-array transceiver is achieved by means of a VLSI adaptive controller implementing the multi-dithering algorithm [3] and adjusting the phase shift introduced to each element of the antenna array at the transmitter. A brief description of the principle of operation is provided below, while further details on the circuit implementation and the performance of the controller can be found in [4].

Multi-dithering relies on parallel perturbation of the variables $\mathbf{u}=\left[u_{i}\right]_{i=1, \ldots, n}=\left[u_{1}, \ldots, u_{n}\right]$ controlling a plant and is applied by superimposing small deterministic and narrowband, typically sinusoidal, dithers of distinct frequencies $\omega_{i}$ on the controls

$$
\tilde{u}_{i}=u_{i}+\alpha \cos \left(\omega_{i} t\right), \alpha \text { small. }
$$

The dithers are translated into gradient dependent fluctuations of a measured metric $J$ that is used to describe the performance of the plant and relative to which optimization of the 
plant is desired

$$
\begin{aligned}
J(\tilde{\mathbf{u}}) & =J\left(\mathbf{u}+\alpha\left[\cos \left(\omega_{i} t\right)\right]_{i=1, \ldots, n}\right) \\
& =J(\mathbf{u})+\alpha(\nabla J(\mathbf{u}))^{\mathrm{T}}\left[\cos \left(\omega_{i} t\right)\right]_{i=1, \ldots, n}+\text { H.O.T. } \\
& =J(\mathbf{u})+\left.\alpha \sum_{i=1}^{n} \frac{\partial J}{\partial u_{i}}\right|_{\mathbf{u}} \cos \left(\omega_{i} t\right)+\text { H.O.T. }
\end{aligned}
$$

Performing parallel synchronous detection (multiplication and lowpass filtering) between the measured metric and each of the dithers, the partial derivative components of the gradient pertinent to each control variable are retrieved

$$
\overline{J(\tilde{\mathbf{u}}) \cos \left(\omega_{i} t\right)}=\left.\frac{\alpha}{2} \frac{\partial J}{\partial u_{i}}\right|_{\mathbf{u}} .
$$

The partial derivatives provide information on the dependence of the performance metric to each individual variable and are supplied in a gradient flow algorithm that leads the plant to optimization

$$
\frac{d u_{i}}{d t}=-G \operatorname{sgn}\left(\left.\frac{\partial J}{\partial u_{i}}\right|_{\mathbf{u}}\right) \text {. }
$$

The gain $G$ can be selected either positive or negative, according to whether minimization or maximization is needed. Retaining and using only the sign information of the partial derivatives for adaptation adds immunity to impulsive noise. The structural level architecture of the adaptive controller is shown in Fig. 2.

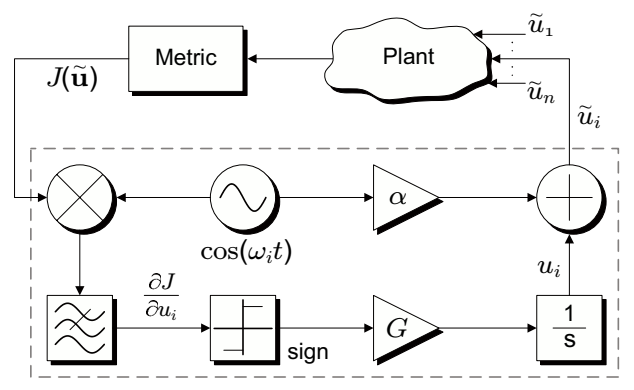

Fig. 2. Structural level architecture of the multi-dithering adaptive control.

\section{Power Maximization AND SynCHROnizATION}

Referring to Fig. 1 and assuming that all elements of the transmitter's antenna array are driven by the same LO operating at a frequency $\omega_{t}$, the total signal at the receiver is

$$
v_{r}(\mathbf{u}(t))=\sum_{i} A_{i} \cos \left(\omega_{t} t+u_{i}(t)+\varphi_{i}\right)
$$

where $A_{i}>0$ is the amplitude of the signal received from the $i$-th antenna element, $u_{i}$ is the phase shift introduced by the adaptive controller, and $\varphi_{i}$ is a phase shift added due to propagation.

The performance metric of the system is constructed by multiplying $v_{r}$ with the output of the RLO and lowpass filtering the product, which results in

$$
J(\mathbf{u}(t))=\sum_{i} A_{i} \cos \left(\Delta \omega \cdot t+u_{i}(t)+\varphi_{i}\right), \Delta \omega=\omega_{t}-\omega_{r}
$$

Note that a quasi-static assumption is made for the setup under consideration with respect to the amplitudes $A_{i}$ and the phase shifts $\varphi_{i}$; phase variations due to the frequency offset between the LOs in the transmitter and the receiver are considered much faster than any topological changes in the wireless link.

The metric $J$ is maximized when all the cosine terms in (2) become 1 . This condition directly implies phase locking since it is satisfied when

$$
\begin{aligned}
\omega_{t} t+u_{i}(t)+\varphi_{i} & =\omega_{r} t+2 \kappa \pi, \kappa \in \mathbb{Z} \Leftrightarrow \\
\Leftrightarrow u_{i}(t) & =\Delta \omega \cdot t-\varphi_{i}+2 \kappa \pi, \kappa \in \mathbb{Z}
\end{aligned}
$$

The adaptation process in the wireless link, according to (3), can be interpreted as phase control in two different timescales: compensation of the slowly varying propagation delays mapped to the parameters $\varphi_{i}$, in order to achieve phase coherency of the transmitted signals at the receiver and thus power maximization; and fast synchronization of the transmitter's reference frequency to that of the receiver.

A question that is directly raised is whether the optimum values for $u_{i}$ dictated by (3) are stable points of the adaptive closed-loop system. Starting from (4) and substituting (2), the state equations of the control system take the form

$$
\frac{d u_{i}}{d t}=G \cdot \operatorname{sgn}\left(A_{i} \sin \left(\Delta \omega \cdot t+u_{i}(t)+\varphi_{i}\right)\right),
$$

where $G<0$ since maximization is required. The equilibrium points of the system include the maximizing values of $u_{i}$ derived in (3), as well as their $\pi$-shifted minimizing counterparts. In order to investigate the stability of the system, (4) is multiplied by $\sin \left(\Delta \omega \cdot t+u_{i}(t)+\varphi_{i}\right)$ which results, after some manipulation, in

$\frac{d}{d t}\left(1-\cos \left(\Delta \omega \cdot t+u_{i}(t)+\varphi_{i}\right)\right)=G\left|\sin \left(\Delta \omega \cdot t+u_{i}(t)+\varphi_{i}\right)\right|$

Function $V\left(u_{i}(t)\right)=1-\cos \left(\Delta \omega \cdot t+u_{i}(t)+\varphi_{i}\right)$ is a positive semi-definite function, with a negative semi-definite derivative and roots equal to the values maximizing $J$. Thus, $V$ is a Lyapunov function and according to Lyapunov's stability theorem [5], the values of $u_{i}$ pointed by (3) will be asymptotically stable equilibrium points.

Using a similar procedure and rationale, and invoking Lyapunov's instability theorem [5], the remaining equilibrium points, that minimize $J$, can be proved to be unstable.

\section{System SETUP}

The general architecture of the implemented wireless setup is shown in Fig. 3. At the transmitter side, the signal from the TLO is split into 4 channels, using a broadband resistive divider. This reference signal is phase-shifted at each channel using a quadrature modulator before driving an antenna element. The in-phase and quadrature components of the modulator are derived by applying the sine and cosine functions, respectively, on the signals $u_{i}$ provided to each channel by the adaptive controller. The 4 channels form an antenna array that communicates with a single antenna receiver.

At the receiver side, a bandpass filter selects a range of frequencies around the reference set by the RLO, that 


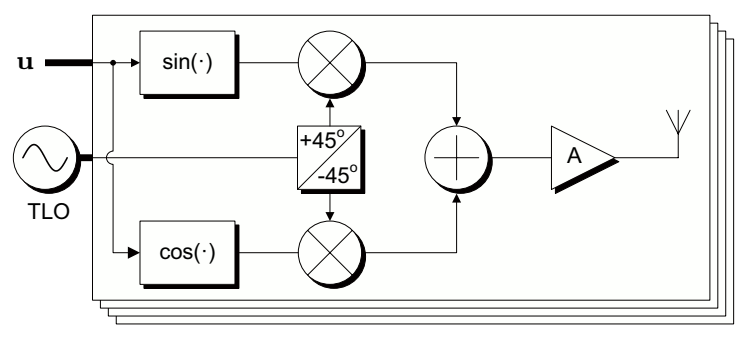

(a)

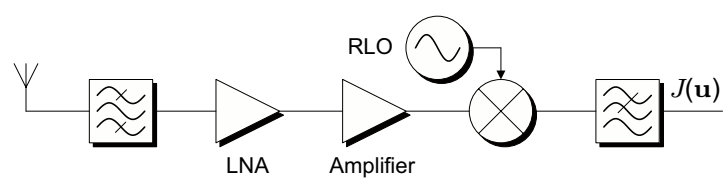

(b)

Fig. 3. Block diagram of the implemented wireless setup: (a) transmitter and (b) receiver.

accommodates the possible expected frequency of the TLO. Note that since a multi-dithering algorithm is employed for adaptive control of the phases, phase modulation of the carrier signal from the TLO will be observed at the receiver. Tones are expected at the frequencies $\omega_{t} \pm \omega_{i}$, where $\omega_{i}$ are the frequencies of the dithers. The dither frequencies are much higher than the expected offset $\Delta \omega$ and therefore their distance from the carrier governs how broad the pass band of the filter is.

Once filtered, the received signal is amplified by an LNA, and sequentially by an optional high gain amplifier. Multiplication with the RLO and lowpass filtering leads to the metric measure of (2) that is fed back to the adaptive controller.

\section{A. Phase Shifting}

Selection of the phase shifting technique used in the architecture was based on the following requirements: control of the phase should be fast, extending to ns response (or, equivalently, a bandwidth of hundreds of $\mathrm{MHz}$ ), the range of attainable phase shifts should cover at least $2 \pi$ and, finally, the phase shifter should be able to operate equally well for a broad range of carrier frequencies. The last specification, although not necessary, takes advantage of the broad operating range of the used multi-dithering controller [4] and allows for a versatile design of the phase locking system, able to accommodate a large range of carrier frequencies.

Among the available designs in literature, passive $L C$ or $R C$ phase shifters (e.g. [6], [7]) suffer from a small range of attainable phases as well as narrowband operation. Phase tunability can be extended by cascading more sections, however, matching between the passive elements is then, usually, required in order to maintain the desired response. The same issues hold for distributed varactor-loaded transmission lines [8] and reflective-type phase shifters [9], [10]. Furthermore, it should be noted that for discrete implementations of such phase shifters the control range of the varactors is usually extremely high, whereas for their integrated versions, the low voltage tuning range comes at the expense of limited resolution and higher phase noise.

Quadrature modulation, although requiring more involved hardware and consuming additional power, offers a viable alternative for phase shifting (e.g. [11]), satisfying at the same time the requirements for fast and broadband operation with a wide phase tuning range. The approach followed in the presented system incorporates analog implementations of the sine and cosine function that map their argument into a phase shift of the carrier when applied to its in-phase and quadrature components.

The design of the sine and cosine circuits involves bipolar transistors and exploits the tanh nature of the transconductance in a BJT differential pair. As shown in [12], summation of adequately offset versions of the hyperbolic tangent results in a close representation of the sine function within a certain range of inputs. This range depends on the number of tanh segments employed (which needs to be odd) and as the limit goes to infinity the sum becomes exactly equal to a sine. For the actual implementation, 3 differential pairs, one with 0 offset and the other 2 with $\pm 3 V_{T}$ (where $V_{T}$ is the thermal voltage) were enough for a single cycle (covering a $2 \pi$ range) sine representation. The circuit topology of the sine function is shown in Fig. 4(a).

The even nature of the cosine function calls for an even number of summed hyperbolic tangents, offset by pairs around 0 . The cosine function was implemented according to Fig. 4(b) by setting the tanh pair offsets at $\pm 1.5 V_{T}$ and $\pm 4.5 V_{T}$. The extra current source accounts for an output offset and brings the mean value of the cosine, within its single cycle representation, at 0 .

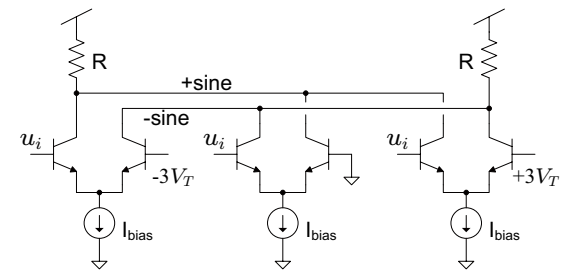

(a)

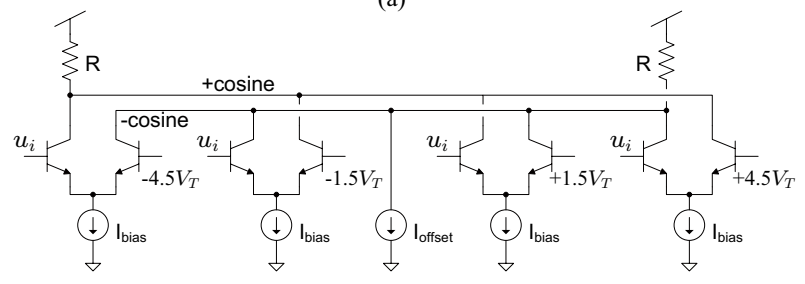

(b)

Fig. 4. Circuit implementation of (a) the sine and (b) the cosine function.

\section{DEMOnSTRATION}

The demonstrated system allows interaction with the user in multiple ways. First, a graphical computer interface allows to set several parameters in the controller and observe their effect on the behavior of the system. Second, the user is able 
to relocate moving parts of the system, inducing variable occlusions and diffraction disturbances, and test the optimization ability of the controller while the transmitter-receiver link is changing. Finally, in order to characterize the bandwidth limit of the system, the user can test the phase locking ability of the loop by varying the frequency offset between transmitter and receiver.

\section{CONCLUSION \\ REFERENCES}

[1] D. N. Loizos, P. P. Sotiriadis, and G. Cauwenberghs, "Multi-channel coherent detection for delay-insensitive model-free adaptive control," in Proc. Int. Symp. Circuits Syst., May 2007, pp. 1775-1778.

[2] L. C. Godara, "Application of antenna arrays to mobile communications. II: Beam-forming and direction-of-arrival considerations," Proc. IEEE, vol. 85, no. 8, pp. 1195-1245, Aug. 1997.

[3] T. R. O'Meara, "The multidither principle in adaptive optics," J. Opt. Soc. Am., vol. 67, no. 3, pp. 306-315, Mar. 1977.

[4] D. N. Loizos, P. P. Sotiriadis, and G. Cauwenberghs, "SiGe BiCMOS 8-channel adaptive controller with sub-microsecond settling," IEEE J. Solid-State Circuits, submitted.

[5] H. K. Khalil, Nonlinear Systems. MacMillan Publishing Company, 1992.

[6] T. M. Hancock and G. M. Rebeiz, "A 12-GHz SiGe phase shifter with integrated LNA," IEEE J. Solid-State Circuits, vol. 53, no. 3, pp. 977983, Mar. 2005.

[7] D. Pache et al., "An improved 3V 2GHz BiCMOS image reject mixer IC," in Proc. Cust. Integr. Circuits Conf. (CICC '95), May 1-4, 1995, pp. 95-98.

[8] A. S. Nagra and R. A. York, "Distributed analog phase shifters with low insertion loss," IEEE Trans. Microw. Theory Tech., vol. 47, no. 9, pp. 1705-1711, Sep. 1999.

[9] H. Zarei, C. T. Charles, and D. J. Allstot, "Reflective-type phase shifters for multiple-antenna transceivers," IEEE Trans. Circuits Syst. I, vol. 54, no. 8, pp. 1647-1656, Aug. 2007.

[10] F. Ellinger, R. Vogt, and W. Bachtold, "Ultracompact reflective-type phase shifter MMIC at C-band with $360^{\circ}$ phase-control range for smart antenna combining," IEEE J. Solid-State Circuits, vol. 37, no. 4, pp. 481-486, Apr. 2002.

[11] K.-J. Koh and G. M. Rebeiz, "0.13- $\mu \mathrm{m}$ CMOS phase shifters for X-, $\mathrm{Ku}-$, and K-band phased arrays," IEEE J. Solid-State Circuits, vol. 42, no. 11, pp. 2535-2546, Nov. 2007.

[12] B. Gilbert, "Circuits for the precise synthesis of the sine function," Electr. Lett., vol. 13, no. 17, pp. 506-508, Aug. 18, 1977. 